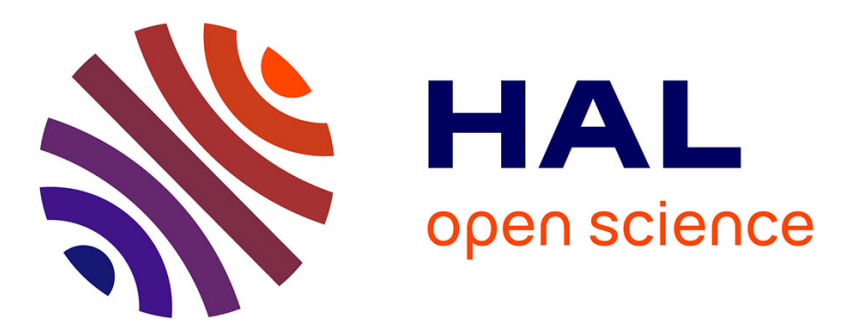

\title{
Third-order many-body perturbation theory applied to Kondo-type dynamics in diluted magnetic semiconductors
}

\author{
Omar Morandi, Paul-Antoine Hervieux
}

\section{- To cite this version:}

Omar Morandi, Paul-Antoine Hervieux. Third-order many-body perturbation theory applied to Kondo-type dynamics in diluted magnetic semiconductors. Physical Review B: Condensed Matter and Materials Physics (1998-2015), 2010, 81, pp.195215. hal-00596699

\section{HAL Id: hal-00596699 \\ https://hal.science/hal-00596699}

Submitted on 30 May 2011

HAL is a multi-disciplinary open access archive for the deposit and dissemination of scientific research documents, whether they are published or not. The documents may come from teaching and research institutions in France or abroad, or from public or private research centers.
L'archive ouverte pluridisciplinaire HAL, est destinée au dépôt et à la diffusion de documents scientifiques de niveau recherche, publiés ou non, émanant des établissements d'enseignement et de recherche français ou étrangers, des laboratoires publics ou privés. 


\title{
Third-order many-body perturbation theory applied to Kondo-type dynamics in diluted magnetic semiconductors
}

\author{
O. Morandi ${ }^{1,2}$ and P.-A. Hervieux ${ }^{2}$ \\ ${ }^{1}$ INRIA Nancy Grand-Est and Institut de Recherche en Mathématiques Avancées, 7 rue René Descartes, F-67084 Strasbourg, France \\ ${ }^{2}$ IPCMS, 23 rue du Loess, F-67037 Strasbourg, France \\ (Received 6 February 2010; revised manuscript received 21 April 2010; published 26 May 2010)
}

\begin{abstract}
Ultrafast magnetization dynamics in diluted magnetic semiconductors is investigated using a model based on the pseudofermion formalism and a third-order many-particle expansion of the exact $p d$ exchange interaction. Dynamical RKKY-like interactions and double-exchange mechanism based on the Kondo interaction emerge naturally from our approach. Our analysis reveals that the many-particle expansion is not generally well defined and an infrared Kondo-like divergence can occur. In particular, the bare polarization propagator fails to converge in the presence of a highly confined hole gas and an enhancement of the ion-hole spin correlation is found for low-dimensional systems. Finally, numerical simulations have been performed on GaMnAs and show that dynamical many-particle correlations play a significant role in the time evolution of the total magnetization.
\end{abstract}

DOI: $10.1103 /$ PhysRevB.81.195215

PACS number(s): 78.20.Ls, 78.30.Fs

\section{INTRODUCTION}

From the pioneering work by Kondo of the anomalous resistivity at low temperature in metals doped with very few magnetic impurities, ${ }^{1,2}$ diagrammatic expansion of the exchange interaction between the magnetic species and the conduction electrons has been a tool of primary importance for the understanding of the microscopical processes underlying the observed phenomena. The apparently simple spinspin exchange interaction postulated by Kondo has revealed a great source of difficulties from both mathematical and physical interpretation points of view. While the original approach followed by Kondo was able to exhibit the primary role played by the spin interaction in the scattering processes between the magnetic impurities and the conduction electrons, the appearance of various divergent terms in the resistivity expansion has early revealed the limits of this approach. Since then, in spite of the great theoretical effort which has been devoted to overcome the technical difficulties and to obtain a clear understanding of the thermodynamical properties of a gas of itinerant fermions interacting with well spatially localized spins, at present, little is known about the dynamical aspects of such systems. With the availability of ultrashort and intense optical sources operating in the femtosecond time scale it is nowadays possible to induce and to investigate nonequilibrium regimes in Kondo-like systems such as, for example, demagnetization processes in diluted magnetic semiconductors (DMS). ${ }^{3}$ In particular, one of the most frequently studied material is the III-V semiconductor gallium arsenide, doped with manganese (GaMnAs), for which the Curie temperature (which is an increasing function of the manganese concentration) can attain the value of $172.5 \mathrm{~K}^{4}$

The ultrafast demagnetization in a DMS is a phenomenon where the Kondo exchange interaction causes a flow of spin polarization and energy from the magnetic-ion impurities to the charge carriers, which is subsequently converted to orbital momentum and then thermalized through spin-orbit and hole-hole interactions. The first attempt to outline a micro- scopical interpretation to the ultrafast spin dynamics for metallic films was given in Ref. 5 and, later, few models have been developed to reproduce quantitatively the observed time-dependent magnetization of DMS. ${ }^{6-8}$ The latter are based on the Markovian hypothesis and the scattering between the holes and the magnetic ions, which is modeled at the first order in the Dyson expansion. At this level of approximation, all dynamical higher-order correlation terms beyond the random-phase approximation are discarded.

Recently, a theoretical study reported in Ref. 9 was addressed to quantify the effect of the dynamical spin correlation in DMS. In their study, the exchange interaction is expanded in terms of the asymptotic parameter $1 / S$, where $S$ is the total spin of the ion. This study has revealed the onset of strong correlation effects beyond the random-phase approximation. Unfortunately, the authors did not provide a signature of these effects on a macroscopical quantity such as the mean magnetization of the sample or the Curie temperature. Within the Kondo lattice framework, in Ref. 10, the spindynamical properties of the system have been derived through the study of the thermal magnon excitations. The closure of the many-particle Green's-function hierarchy is resolved by the application of the Tyablikov decoupling hypothesis. A different approach is proposed in Ref. 11 where a method for performing atomistic spin-dynamics simulations is presented. In their approach the ions are treated as paramagnetic atomic moments and their evolution is given by the phenomenological Landau-Lifshitz-Gilbert equation. The energy dissipation of the system is described in terms of a single ad hoc damping parameter. The linking with the microscopical dynamics is provided by the Langevin equation. The spatial-dependent exchange parameter included in the spin dynamics simulations are calculated within the densityfunctional theory and are strongly direction dependent. In Ref. 12, the magnetism evolution in a DMS has been investigated by means of atomistic spin-dynamics simulations where the interatomic exchange interaction is calculated selfconsistently. In analogy with the Born-Oppenheimer approximation, this procedure relies on the adiabatic approxi- 
mation. The time evolution of the orientations of the atomic spins corresponds to the slow nuclear motion and the relaxation of the electron system toward the instantaneous directions of the magnetic moments is related to the fast time evolution. Within this approximation, the equations of motion for the atomic spins and for the electronic degrees of freedom may be solved separately. Contrary to the model of Ref. 12, in the present work the dynamical effects under a strong laser excitation are carefully reproduced, by allowing the time evolution of the ion-hole spin correlations and the off-diagonal components of the electronic density matrix to take place at the same time scale.

In order to obtain a proper description of the spin-spin dynamical correlation effects, we derive in this work an $a b$ initio model. A special attention has been paid to relate the microscopic correlation effects to the time evolution of macroscopic quantities of the material like the mean magnetization. Our method is able to treat the dynamical particleparticle correlations beyond the mean-field approximation, allowing to a more accurate description of the magnetization dynamics. It is based on the application of the Abrikosov pseudofermion formalism and the inclusion of terms up to the third order in the expansion of the Kondo interaction. In particular, the present model extends the results derived in Ref. 8 where only second-order terms had been retained. The Abrikosov approach is particularly suitable to treat ion with high spin number such as manganese. Indeed, it is well known that, in the second quantization formalism, almost all the standard theorems concerning the many-particle expansion of the contour-ordered operators like the Wick theorem for the Green's function are based on elementary commutation rules for the creation and annihilation operators. The lack of simple properties of the commutation relations for the magnetic-ion spin operators (with spin number greater than 1/2) prevents the use of a systematic expansion procedure such as the Feynman diagram expansion to obtain high-order interaction terms. In order to circumvent this technical difficulty, a theoretical study of the Kondo-type interaction needs a special treatment. Abrikosov proposed an approach based on the use of fictive particles (pseudofermions) which formally preserve the standard commutation rules of spin-1/2 particles. ${ }^{13}$ Unfortunately, this formalism introduces unphysical states in the Hilbert space of the magnetic ions for which the impurity sites are allowed to be multiply occupied. Therefore, a suitable limit procedure is required in order to recover the correct physical description of the magnetic species.

The paper is organized as follows: in Sec. II A a brief description of the pseudo-fermion formalism and its main properties are given. Special emphasis is put on the derivation of a set of rules. These latter are needed to evaluate the physically correct Green's function of the system using the many-body expansion procedure applied to the interaction between the fictitious Abrikosov particles. In Sec. II B the equations of motion of the mean magnetization for a system composed of two different types of spin particles and interacting via a Kondo-type scattering term are derived. In Sec. II C, the polarization propagator (which is the most interesting quantity in our model) is evaluated up to the third order in the interaction and its properties are discussed. As an ap- plication, in Sec. III we have applied our model to the study of the out-of-equilibrium magnetization dynamics in a DMS excited by a laser pulse. Numerical results are presented and the role played by the order of the interaction is discussed. Finally, the paper is ended with concluding remarks.

\section{MODEL}

\section{A. Pseudofermion limit}

We describe the time evolution of two different populations of particles (hereafter the symbols $h$ and $M$ are used to represent the holes and the magnetic ions, respectively) in the second quantization formalism. We denote by $a_{k, s}^{\dagger}\left(a_{k, s}\right)$ and $b_{\eta, m}^{\dagger}\left(b_{\eta, m}\right)$ the creation (annihilation) operators of a hole having a spin $s$ and a quasimomentum $k$ and a pseudofermion ion with spin $m$ and spatial position $R_{\eta}$, respectively. In particular, the $b$ operator denotes the Abrikosov pseudofermion operator which gives the correct physical states provided that one and only one particle can be found in each ion site, i.e., $\hat{n}_{\eta}=\sum_{m=-S^{M}}^{S^{M}} \hat{n}_{\eta}^{m}=1$, where $\hat{n}_{\eta}^{m}=b_{\eta, m}^{\dagger} b_{\eta, m} \forall \eta$ and $S^{M}$ $=5 / 2$. The time evolution of the system is governed by the Hamiltonian

$$
\mathcal{H}=\sum_{k, s} \varepsilon_{k, s} a_{k, s}^{\dagger} a_{k, s}+\mathcal{H}_{p d} .
$$

In the parabolic band approximation the kinetic energy of the holes reads $\varepsilon_{k, s}=E^{h}-\hbar^{2} k^{2} / 2 m^{*}$, where $E^{h}$ is the valence band edge and $m^{*}$ is the effective mass of the hole. The Kondolike exchange interaction $\mathcal{H}_{p d}$ is given by

$$
\begin{gathered}
\mathcal{H}_{p d}=\sum\left(b_{\mathbf{m}^{\prime}}^{\dagger} b_{\mathbf{m}} a_{\mathbf{s}^{\prime}}^{\dagger} a_{\mathbf{s}}\right) \mathcal{V}_{\mathbf{m}^{\prime}, \mathbf{m}, \mathbf{s}^{\prime}, \mathbf{s}}, \\
\mathcal{V}_{\mathbf{m}^{\prime}, \mathbf{m}, \mathbf{s}^{\prime}, \mathbf{s}}=\frac{\gamma_{p d}}{V} \delta_{\eta, \eta^{\prime}} e^{i\left(R \eta^{k-R} R_{\left.\eta^{\prime} k^{\prime}\right)} \mathbf{J}_{m^{\prime}, m} \cdot \boldsymbol{\sigma}_{s^{\prime}, s},\right.}
\end{gathered}
$$

where the sum is extended over all indices and the compact notations $\mathbf{m} \equiv(\eta, m), \mathbf{s} \equiv(k, s)$ have been employed. $\gamma_{p d}$ is the $p$ - $d$ coupling constant, $V$ is the volume of the system, and $\boldsymbol{\sigma}, \boldsymbol{J}$, are the spin matrices related to the holes and the ions, respectively. To recover the correct ion-hole exchange interaction (where the ion sites are singly occupied), following Refs. 13 and 14 we add a "fictitious" ionic chemical potential $\mathcal{H}^{\lambda}=\Sigma_{\eta} \lambda_{\eta} \hat{n}_{\eta}$ to the original Hamiltonian and we let $\lambda_{\eta}$ go to infinity at the end of the calculation. The grand-canonical expectation value of a generic operator $\mathcal{A}$ in the pseudofermion space is defined in the usual way,

$$
\begin{aligned}
\langle\mathcal{A}\rangle_{\lambda}^{P F} & =\frac{1}{\mathcal{Z}_{\lambda}^{P F}} \operatorname{Tr}\left\{\rho_{\mathcal{H}} \exp \left(-\beta \sum_{\eta} \lambda_{\eta} \hat{n}_{\eta}\right) \mathcal{A}\right\} \\
& =\frac{1}{\mathcal{Z}_{\lambda}^{P F}} \sum_{\left\{n_{\eta^{m}}^{m_{r}}\right.}\left\langle n_{\eta}^{m}\left|\rho_{\mathcal{H}} \exp \left(-\beta \sum_{\eta} \lambda_{\eta^{n}} \hat{n}_{\eta}\right) \mathcal{A}\right| n_{\eta}^{m}\right\rangle,
\end{aligned}
$$

where $\mathcal{Z}_{\lambda}^{P F}=\operatorname{Tr}\left\{\rho_{\mathcal{H}} \exp \left(-\beta \Sigma_{\eta} \lambda_{\eta} \hat{n}_{\eta}\right)\right\}, \quad \rho_{\mathcal{H}}=e^{-\beta \mathcal{H}}, \quad$ and $\quad \beta$ $=1 / k_{B} T$, with $k_{B}$ the Boltzmann constant and $T$ the temperature. $\left\{n_{\eta}^{m}\right\}_{r}=\left\{n_{1}^{1}, \ldots, n_{r}^{\left(2 S^{M}+1\right)}\right\}$ denotes all possible occupation numbers $n_{\eta}^{k}(=0$ or 1$)$ for $r$ ion sites. By following Coleman, ${ }^{14}$ the correct expectation value of the operator $\mathcal{A}$ is obtained by using the limit 


$$
\langle\mathcal{A}\rangle^{F}=\frac{1}{\mathcal{Z}^{F}} \lim _{\left\{z_{\eta}\right\} \rightarrow 0} \frac{\partial^{r}\left[\langle\mathcal{A}\rangle_{\lambda}^{P F} \mathcal{Z}_{\lambda}^{P F}\right]}{\partial z_{1} \cdots \partial z_{r}},
$$

where $\mathcal{Z}^{F}=\lim _{\left\{z_{\eta}\right\} \rightarrow 0}\left(\partial^{r} \mathcal{Z}^{P F}\right) /\left(\partial z_{1} \cdots \partial z_{r}\right)$ and $z_{\eta}=e^{-\beta \lambda_{\eta}}$. The expectation value of a generic operator $\langle\mathcal{A}\rangle_{\lambda}^{P F}$ defined on the Abrikosov pseudofermion space can be expanded in the usual way by means of the Wick theorem. As a final result, the Langreth theorem for the time-ordered product ensures that the expectation value can be expressed as a product of simpler single-particle Green's functions. As a final result $\langle\mathcal{A}\rangle_{\lambda}^{P F}$ can be expanded as follows:

$$
\langle\mathcal{A}\rangle_{\lambda}^{P F}=\sum_{\xi} \prod_{\eta \in X} \mathbf{A}_{\eta}^{\xi}\left(\mathcal{G}_{\eta}^{>}\right)^{g_{\eta}^{\xi}}\left(\mathcal{G}_{\eta}^{<}\right)^{l_{\eta}^{\xi}}
$$

where the pseudofermion (less-than $\mathcal{G}_{\eta}^{<}$and greater-than $\mathcal{G}_{\eta}^{>}$) Green's functions are defined as in Eq. (4) with $\mathcal{A}$ $=b_{\eta, m}^{\dagger} b_{\eta, m}\left(\mathcal{A}^{\dagger}=b_{\eta, m} b_{\eta, m}^{\dagger}\right)$. For the sake of simplicity, the in$\operatorname{dex} m$ in the Green's functions is not explicitly mentioned. $\mathbf{A}_{\eta}^{\xi}$ are the expansion coefficients of $\left\langle\mathcal{A}_{w}\right\rangle_{\lambda}^{P F}$ on the monomial $\mathcal{G}_{\eta}^{\eta} \mathcal{G}_{\eta}^{<}$for a given couple of the exponents $g_{\eta}^{\xi}, l_{\eta}^{\xi}$ and for which, the explicit form depends on the details of the interaction. $X$ denotes the different ion sites appearing in the $\mathcal{A}_{w}^{\xi}$ expansion. The fermionic limit of Eq. (5) applied to Eq. (6) leads to a very simple expression. Indeed, we have

$$
\left\langle\mathcal{A}_{w}\right\rangle^{F}=\sum_{\xi} \prod_{\eta \in X} \mathbf{A}_{\eta}^{\xi} \delta_{l_{\eta}^{\xi}},^{1} \mathfrak{G}_{\eta}^{<},
$$

where $\mathfrak{G}_{\eta}^{<}=\left(1 / \mathcal{Z}^{F}\right) \lim _{\left\{z_{\eta}\right\} \rightarrow 0}\left(\partial^{r}\left[\mathcal{G}_{\eta}^{<} \mathcal{Z}_{\lambda}^{P F}\right]\right)\left(/ \partial z_{1} \cdots \partial z_{r}\right)$ is the physically correct Green's function in the fermionic limit. A detailed derivation of Eq. (7) is given in Ref. 15. The above expression shows that, in the Abrikosov-Coleman formalism, the fermionic limit can be easily recovered through the usual many-particle expansion applied to the pseudofermion Green's ion propagator. In particular, Feynman diagrams containing more than one single less-than Green's function for each ion site vanish in the pseudofermion limit and the greater-than Green's functions reduce to identity. Differing from the original Abrikosov approach, the Coleman limit procedure allows us to recover the expression of the manyparticle expansion in the physical space directly in terms of the corresponding Green's functions $\mathfrak{G}$, with their explicit expressions being not needed. Therefore, this procedure may be successfully applied to describe dynamical properties of Kondo-type systems (such as a DMS) for which, nonequilibrium Green's functions cannot be approximated by multipole functions in the energy representation.

\section{B. Equations of motion}

The time evolution of the mean magnetization of a DMS can be directly related to the evolution of the hole and ion polarization propagators which are defined as follows (see, for example, Ref. 16):

$$
i \Pi_{\mathbf{m}, \mathbf{s}, \mathbf{m}^{\prime}, \mathbf{s}^{\prime}}=-\lim _{t^{\prime} \rightarrow t^{+}} \mathcal{G}_{\mathbf{m}, \mathbf{s}, \mathbf{m}^{\prime}, \mathbf{s}^{\prime}}^{I I}\left(t, t, t^{\prime}, t^{\prime}\right),
$$

where the two-particle Green's function $\mathcal{G}^{I I}$ is given by

$$
\begin{aligned}
\mathcal{G}_{\mathbf{m}, \mathbf{s}, \mathbf{m}^{\prime}, \mathbf{s}^{\prime}}^{I I}\left(t, t_{1}, t^{\prime}, t_{1}^{\prime}\right)= & -\frac{1}{\mathcal{Z}_{\lambda}^{P F}} \operatorname{Tr}\left\{\exp \left(-\beta \sum_{\eta} \lambda_{\eta^{n}} \hat{n}_{\eta}\right)\right. \\
& \left.\times \mathcal{T}\left[b_{\mathbf{m}}(t) a_{\mathbf{s}}\left(t_{1}\right) b_{\mathbf{m}^{\prime}}^{\dagger}\left(t^{\prime}\right) a_{\mathbf{s}^{\prime}}^{\dagger}\left(t_{1}^{\prime}\right)\right]\right\},
\end{aligned}
$$

where $\mathcal{T}$ denotes the usual time-ordering operator. The annihilation and creation operators are expressed in the Heisenberg's formalism as $b_{\mathbf{m}}(t)=e^{(i / \hbar) \mathcal{H}\left(t-t_{0}\right)} b_{\mathbf{m}} e^{-(i / \hbar) \mathcal{H}\left(t-t_{0}\right)}$. The time-evolution equation for the mean densities, $n_{s}$ $\equiv\left(1 / N^{h}\right) \Sigma_{k}\left\langle a_{k, s}^{\dagger} a_{k, s}\right\rangle$ and $\mathfrak{n}_{m} \equiv\left(1 / N^{M}\right) \Sigma_{\eta}\left\langle b_{\eta, m}^{\dagger} b_{\eta, m}\right\rangle$, can be easily derived from the Heisenberg equations of motion, ${ }^{8}$

$$
\begin{gathered}
i \hbar \frac{d n_{s}}{d t}=\frac{1}{N^{h}} \sum_{m} \mathcal{W}_{s, m}, \\
i \hbar \frac{d \mathfrak{n}_{m}}{d t}=\frac{1}{N^{M}} \sum_{s} \mathcal{W}_{s, m}, \\
\mathcal{W}_{s, m}=\frac{\gamma_{p d}}{V} \sum_{s^{\prime}, m^{\prime}} \Theta_{m^{\prime}, m, s^{\prime}, s} \sum_{k, k^{\prime}, \eta, \eta^{\prime}} \delta_{\eta, \eta^{\prime}}\left(e^{i R \eta^{\left(k-k^{\prime}\right)}} \Pi_{\mathbf{m}^{\prime}, \mathbf{m}, \mathbf{s}^{\prime}, \mathbf{s}}\right. \\
\left.-e^{-i R_{\eta^{\prime}}\left(k-k^{\prime}\right)} \Pi_{\mathbf{m}, \mathbf{m}^{\prime}, \mathbf{s}, \mathbf{s}^{\prime}}\right),
\end{gathered}
$$

where $\Theta_{m^{\prime}, m, s^{\prime}, s}=\mathbf{J}_{m^{\prime}, m} \cdot \boldsymbol{\sigma}_{s^{\prime}, s}$ and $N^{h}, N^{M}$ are the total density of holes and ions, respectively. We calculate the diagrammatic expansion of the polarization propagator up to the third order in the interaction $\mathcal{V}$. Within the first order, the static spin polarization is taken into account at a mean-field level. The second-order term represents the exchange scattering process between the ions localized at the $R_{\eta}$ positions and the itinerant holes within the same Wigner-Seitz cell. At this level of approximation, the spin flux between the two populations of particles is modeled via detailed balance equations. These contributions have been analyzed in detail in a series of works. ${ }^{7,8,17}$ Despite the fact that the second-order approximation is able to qualitatively reproduces the observed dynamical behavior of the total magnetization in a DMS, recent works have revealed that ground-state properties of the DMS cannot be properly reproduced within a first-order Zener mean-field model. ${ }^{18}$ Therefore, it is natural to expect a corresponding non-negligible correction on the dynamical properties and, in particular, on the time evolution of the total magnetization. Within the mean-field approximation magnetization fluctuations are neglected and generally the theoretical models lead to an overestimation of the magnitude of the Curie temperature. In Ref. 19 it is shown that doubleexchange and superexchange mechanisms can play an important role on the static polarization of a DMS. According to these considerations the present work is addressed to the study of the third-order exchange contributions and its influence on the time evolution of the total magnetization in a DMS. We will give a physical interpretation of the different terms which appear in the model. Finally, analogies with similar processes derived within different theoretical frameworks will be also discussed. 

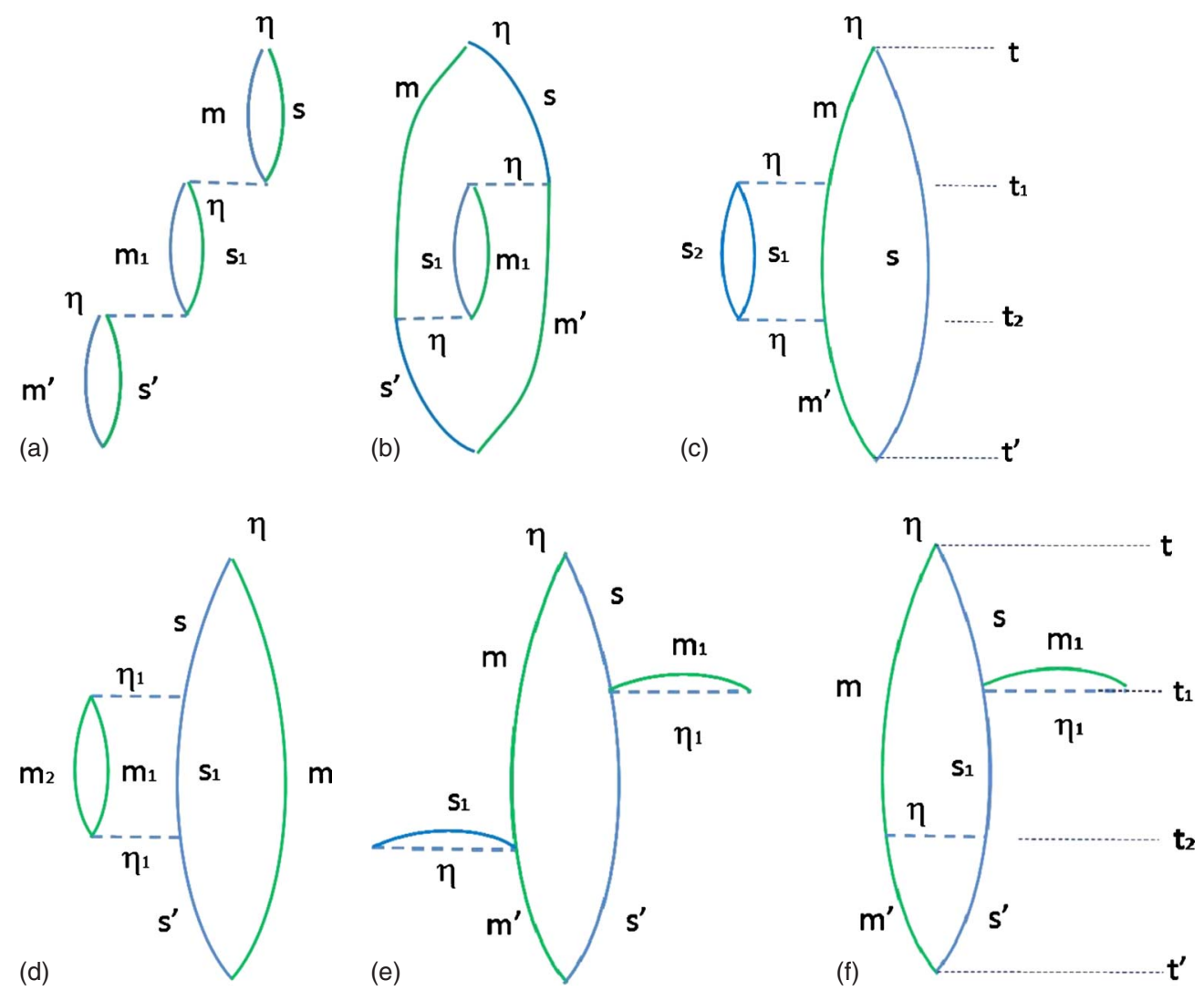

FIG. 1. (Color online) Third-order diagrammatic expansion of the hole-Mn spin-exchange interaction.

\section{Polarization propagator: Physical interpretation and renormalization}

The nonvanishing third-order Feynman diagrams of $\Pi$ are represented in Fig. 1 and provide a clear physical interpretation of the third-order ion-hole scattering processes. The green-continuous (blue-continuous) lines represent the pseudoparticle (hole) propagator and the dashed lines denote the exchange interaction. Diagrams in Figs. 1(a)-1(c) describe the multiple exchange interaction between the holes and an ion localized at a single site (denoted by $\eta$ ), while the second group of diagrams [Figs. 1(d)-1(f)] represent the exchange interactions involving two different ion sites. In the latter, an excited state containing a quantum entanglement between the ion and the hole spin is initially created at $R_{\eta}$ and subsequently undergoes a further spin-exchange interaction at a different site $R_{\eta^{\prime}}$ : the itinerant character of the hole Bloch wave establishes a hole-mediated correlation between two different ion sites. Since the ion does not possess kinetic energy, any movement from its spatial position is forbidden and consequently the bare ion Green's function is diagonal with respect to the variable $R_{\eta}$. Thus, the subdiagram where all the interaction vertices are connected by an ion propagator represent local-in-space processes (see, for example, Ref. 14 for a general discussion of the Kondo-Feynman diagram method). Going more into details, the diagram in Fig. 1(d) (similar considerations apply to the other diagrams) describes dynamical polarization effects of the holes-ions system where, at the time $t$, a spin entanglement is created at $R_{\eta}$ between a hole (with spin down, for example) and an ion pseudoparticle. Subsequently, the hole travels toward the site $R_{\eta_{1}}$ where, at $t=t_{1}$ it interacts with the ion located there, raising its spin and a hole-ion excited state is created. Recombination arises at $t=t_{2}$ where the hole lowers its spin again. Finally, the initial unexcited state is recovered at $t$ $=t^{\prime}$ where the hole interacts with the ion at $R_{\eta}$. The ion-hole bubble mediates the carrier-induced ion spin couplings, and the oscillatory long-range part of the spin interaction is reproduced by a dynamical out-of-equilibrium RKKY-like phenomenon. Usually it is postulated that the RKKY interaction plays the major role in the ion-ion hole-mediated interaction and the exchange interaction is written as $\mathcal{H}_{p d}$ $=\sum_{i j} J_{i j}^{R K K Y} \mathbf{S}_{i} \cdot \mathbf{S}_{j}$, where $\mathbf{S}_{i}$ is the ion spin at site $R_{i}$ and $J_{i j}^{R K K} \stackrel{i j}{=} J_{0} r^{-4}\left[\sin \left(2 k_{F} r\right)-2 k_{F} r \cos \left(2 k_{F} r\right)\right] . J_{0}$ is the local Zener coupling parameter and $k_{F}$ is the Fermi momentum of the holes. ${ }^{18,20}$ In order to derive the RKKY interaction the hole degrees of freedom are integrated out, so that the hole distribution plays essentially a rather minor role in the polarization processes and enters in the model only through the mean Fermi momentum (or the susceptibility). On the contrary, in our approach, the RKKY interaction emerges naturally from the pseudofermion expansion of the exchange interaction and the associated formalism is able to describe strong-outequilibrium hole distribution as it is the case after a pulse laser excitation. This statement will be confirmed in the following where we present an explicit evaluation of the polarization propagators associated with the diagrams in Figs. 1(b)-1(d): the hole-mediated interaction depends on the energy integral of the out-of-equilibrium hole distribution which dynamically evolves during the demagnetization pro- 
cesses. In Ref. 18 it is shown that spin-interacting models based on the mean-field approximation are not able to accurately model the static characteristics and, in particular, they overestimate both the Curie temperatures and the stability of ferromagnetic materials as a function of the charge-carrier density. Therefore, models able to take in account phenomena occurring beyond the mean-field approximation can provide a deeper insight of the nontrivial time evolution of the total magnetization of a magnetic semiconductor.

In what follows, we give the details of the calculation of the contributions to the equation of motion (10) arising from the diagrams in Figs. 1(b)-1(d) (the other terms are treated similarly). We have

$$
\begin{aligned}
& \Pi_{\mathbf{m}, \mathbf{s}, \mathbf{m}^{\prime}, \mathbf{s}^{\prime}}^{b}=-\frac{1}{2} \sum_{\mathbf{m}_{1}, \mathbf{s}_{1}} \mathcal{V}_{\mathbf{m}_{1}, \mathbf{m}^{\prime}, \mathbf{s}, \mathbf{s}_{1}} \mathcal{V}_{\mathbf{m}, \mathbf{m}_{1}, \mathbf{s}_{1}, \mathbf{s}^{\prime}} \sum_{(i, j, k)} \mathcal{I}_{i, j, k}, \\
& \mathcal{I}_{i, j, k}=\int \widetilde{\mathcal{G}}_{\mathbf{m}, \mathbf{s}^{\prime}}^{i}\left(t, t_{2}\right) \widetilde{\mathcal{G}}_{\mathbf{m}_{1}, \mathbf{s}_{1}}^{j}\left(t_{2}, t_{1}\right) \widetilde{\mathcal{G}}_{\mathbf{m}^{\prime}, \mathbf{s}}^{k}\left(t_{1}, t^{+}\right) d t_{1} d t_{2},
\end{aligned}
$$

where $(i, j, k)=(<, a, a) ;(r,<, a) ;(r, r,<)$ and the Langreth theorem has been used. To ease notation we have defined $\widetilde{\mathcal{G}}_{\mathbf{m}, \mathbf{s}}\left(t, t_{1}\right) \equiv{ }^{M} \mathcal{G}_{\mathbf{m}, \mathbf{m}}\left(t, t_{1}\right)^{h} \mathcal{G}_{\mathbf{s}, \mathbf{s}}\left(t_{1}, t\right)$, where ${ }^{M} \mathcal{G}_{\mathbf{m}, \mathbf{m}}$ and ${ }^{h} \mathcal{G}_{\mathbf{s}, \mathbf{s}}$ denote the pseudofermion Green's function for the holes and for the ions, respectively. Explicitly we have

$$
\begin{aligned}
{ }^{M} \mathcal{G}_{\mathbf{m}, \mathbf{m}}\left(t_{2}, t_{1}\right)= & \frac{1}{\mathcal{Z}_{\lambda}^{P F}} \\
& \times \operatorname{Tr}\left\{\exp \left(-\beta \sum_{\eta} \lambda_{\eta} \hat{n}_{\eta}\right) \mathcal{T}\left[b_{\mathbf{m}}\left(t_{2}\right) b_{\mathbf{m}}^{\dagger}\left(t_{1}\right)\right]\right\},
\end{aligned}
$$

and since the hole Green's function does not depend on the ion degrees of freedom its limit $\lambda \rightarrow \infty$ trivially leads to the usual definition of the Green's function,

$$
{ }^{h} \mathcal{G}_{\mathbf{s}, \mathbf{s}}\left(t_{2}, t_{1}\right)=\frac{\left\langle\Psi\left|\mathcal{T}\left[a_{\mathbf{s}}\left(t_{2}\right) a_{\mathbf{s}^{\prime}}^{\dagger}\left(t_{1}\right)\right]\right| \Psi\right\rangle}{\langle\Psi \mid \Psi\rangle}
$$

where $\Psi$ denotes the many-particle wave function containing only the hole degrees of freedom. The superscripts $a(r)$ and $<(>)$ denote, respectively, the usual advanced (retarded) and less- (greater-) than functions and whose definition is given as usually in analogy with Eqs. (13) and (14). Equation (12) is of the form

$$
\mathcal{C}\left(t, t^{\prime}\right)=\int_{-\infty}^{\infty} \mathcal{A}\left(t, t_{2}\right) \mathcal{B}\left(t_{2}, t^{\prime}\right) d t_{2} .
$$

We define the temporal variables $T=\left(t+t^{\prime}\right) / 2$ and $\tau=t-t^{\prime}$. We have the following gradient expansion of $\hat{\mathcal{C}}(\omega, T)$ $\equiv \int \mathcal{C}(\tau, T) e^{i \tau \omega} d \tau($ Ref. 21):

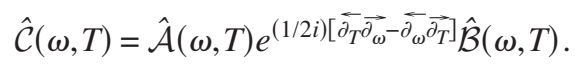

If we apply the previous formula to Eq. (12) retaining only the lowest-order contribution of the gradient expansion we obtain

$$
\begin{aligned}
\mathcal{I}_{i, j, k}= & \iint \tilde{\mathcal{G}}_{\mathbf{m}, \mathbf{s}^{\prime}}^{i}\left(t-t_{2}, T\right) \\
& \times \widetilde{\mathcal{G}}_{\mathbf{m}_{1}, \mathbf{s}_{1}}^{j}\left(t_{2}-t_{1}, T\right) \widetilde{\mathcal{G}}_{\mathbf{m}^{\prime}, \mathbf{s}}^{k}\left(t_{1}-t, T\right) d t_{1} d t_{2} .
\end{aligned}
$$

Following the pseudofermion limit procedure outlined in Sec. II A, in the limit $\lambda \rightarrow \infty$ the only nonvanishing contribution of $\mathcal{I}_{r, r,<}$ is $\left(\mathcal{I}_{<, a, a}\right.$ and $\mathcal{I}_{r,<, a}$ can be treated similarly)

$$
\begin{aligned}
\lim _{\lambda \rightarrow \infty} \mathcal{I}_{r, r,<}= & \int_{-\infty}^{t} \int_{-\infty}^{t_{2}} \tilde{\mathcal{G}}_{\mathbf{m}, \mathbf{s}^{\prime}}^{>}\left(t-t_{2}, T\right) \widetilde{\mathcal{G}}_{\mathbf{m}_{1}, \mathbf{s}_{1}}^{>}\left(t_{2}-t_{1}, T\right) \\
& \times \widetilde{\mathcal{G}}_{\mathbf{m}^{\prime}, \mathbf{s}}^{<}\left(t_{1}-t, T\right) d t_{1} d t_{2} .
\end{aligned}
$$

In the quasiparticle approximation $\tilde{\mathcal{G}}_{\mathbf{m}, \mathbf{s}^{\prime}}^{>}\left(t-t_{2}, T\right)$ $\simeq \widetilde{\mathcal{G}}_{\mathbf{m s}^{\prime}}^{>}(T) e^{-i\left(E_{\mathbf{m}}+E_{\mathbf{s}^{\prime}}\right)\left(t-t_{2}\right)}$, where $E_{\mathbf{m}}\left(E_{\mathbf{s}}\right)$ is the eigenvalue of the ion (hole) state described by the quantum numbers $\mathbf{m}(\mathbf{s})$. In this case the previous formula reduces to

$$
\begin{aligned}
\lim _{\lambda \rightarrow \infty} \mathcal{I}_{r, r,<} \simeq & \widetilde{\mathcal{G}}_{\mathbf{m}, \mathbf{s}^{\prime}}^{>}(T) \tilde{\mathcal{G}}_{\mathbf{m}_{1}, \mathbf{s}_{1}}^{>}(T) \tilde{\mathcal{G}}_{\mathbf{m}^{\prime}, \mathbf{s}}^{<}(T)\left(\frac{i}{\Delta_{\mathbf{m}^{\prime}, \mathbf{s}^{\prime}}^{\mathbf{m}^{\prime}, \mathbf{s}}+i \chi}\right) \\
& \times\left(\frac{i}{\Delta_{\mathbf{m}_{1}, \mathbf{s}_{1}}^{\mathbf{m}^{\prime}, \mathbf{s}}+i \chi}\right),
\end{aligned}
$$

with

$$
\Delta_{\mathbf{m}, \mathbf{s}}^{\mathbf{m}^{\prime}, \mathbf{s}^{\prime}}=E_{\mathbf{m}^{\prime}}-E_{\mathbf{m}}+E_{\mathbf{s}^{\prime}}-E_{\mathbf{s}},
$$

and where we have used the Dirac formula

$$
\int_{-\infty}^{t} e^{i(\Delta+i \chi)\left(t-t_{1}\right)} d t_{1}=\frac{i}{\Delta+i \chi}=\pi \delta(\Delta)+i \mathcal{P} \frac{1}{\Delta}
$$

for a small $\chi$ and $\mathcal{P}$ denotes the principal value. We are interested in studying the properties of the time-dependent polarization propagator: for the sake of clearness we report here the explicit form of the single-site diagram [Fig. 1(b)] and the two-site diagram [Fig. 1(d)] (denoted by $\Pi^{b}$ and $\Pi^{d}$, respectively). After a rather cumbersome algebra we obtain

$$
\begin{aligned}
\sum_{\eta, \eta^{\prime}} & \delta_{\eta, \eta^{\prime}} \Pi_{\mathbf{m}^{\prime}, \mathbf{m}, \mathbf{s}^{\prime}, \mathbf{s}}^{b} e^{i R} \eta^{\left(k-k^{\prime}\right)} \\
& =-i 2 \pi \gamma_{p d^{2}}^{2} N^{M} \sum_{m_{1}, s_{1}} \Theta_{m, m_{1}, s_{1}, s^{\prime}} \Theta_{m_{1}, m^{\prime}, s, s_{1}} \mathfrak{n}_{m^{\prime}}{ }^{h} \mathcal{G}_{\mathbf{s}^{\prime}}^{<{ }^{h}} \mathcal{G}_{\mathbf{s}}^{>} \mathcal{F}_{s_{1}}^{<} \\
& \times\left(\Delta_{m_{1}, s_{1}}^{m^{\prime}, s}+E_{k}\right) \delta\left(E_{k}-E_{k^{\prime}}+\Delta_{m, s^{\prime}}^{m^{\prime}, s}\right), \\
\sum_{\eta, \eta^{\prime}} & \delta_{\eta, \eta^{\prime}} \Pi_{\mathbf{m}^{\prime}, \mathbf{m}, \mathbf{s}^{\prime}, \mathbf{s}^{\prime}}^{d} e^{i R_{\eta^{\prime}}\left(k-k^{\prime}\right)} \\
& =i 4 \pi^{2} \gamma_{p d}^{2}\left(N^{M}\right)^{2} \mathfrak{n}_{m} \delta_{m, m^{\prime}} \sum_{m_{1}, s_{1}, m_{2}} \Theta_{m_{1}, m_{2}, s, s_{1}} \Theta_{m_{2}, m_{1}, s_{1}, s^{\prime}} \mathcal{Q},
\end{aligned}
$$

where

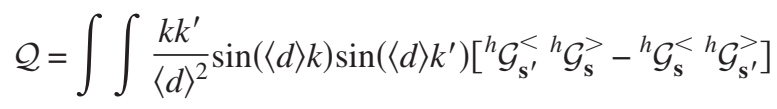




$$
\times\left[\mathfrak{n}_{m_{1}} \mathcal{F}_{s_{1}}^{>}(\widetilde{\Delta})-\mathfrak{n}_{m_{2}} \mathcal{F}_{s_{1}}^{<}(\tilde{\Delta})\right] \delta\left(E_{\mathbf{s}^{\prime}}-E_{\mathbf{s}}\right) d k d k^{\prime},
$$

with

$$
\mathcal{F}_{s_{1}}^{\gtrless}(\xi)=\mathcal{P} \int_{0}^{\infty} \frac{f_{s_{1}}^{\gtrless}\left(\xi-E_{1}\right) \rho\left(E_{1}\right)}{\xi-E_{1}} d E_{1} .
$$

Here, $\widetilde{\Delta}=E_{s_{1}}+E_{m_{1}}+E_{m_{2}}-E_{\mathbf{s}}$ and $\langle d\rangle$ is the mean distance between two adjacent ions, and to ease notation we have defined $f_{s}^{\gtrless}(\xi)={ }^{h} \mathcal{G}_{k=\sqrt{2 m^{*} \xi, s}}^{\gtrless}$, where ${ }^{h} \mathcal{G}_{\mathbf{s}} \equiv{ }^{h} \mathcal{G}_{\mathbf{s}, \mathbf{s}}$ and $\rho$ is the density of states. In order to derive the previous equations we have used the limit of disordered ion distribution. Explicitly, for a given function $h$ we use the following approximation:

$$
\sum_{\eta, \eta_{1}} h\left(\left|R_{\eta_{1}}-R_{\eta}\right|\right) \mathcal{G}_{\eta} \mathcal{G}_{\eta_{1}} \simeq h(\langle d\rangle) \sum_{\eta} \mathcal{G}_{\eta} \sum_{\eta_{1}} \mathcal{G}_{\eta_{1}} .
$$

Similarly to the usual RKKY interaction, in $\Pi^{d}$ we note the appearance of the hole momentum in the dynamical and spatially dependent coherent modulation of the ion-ion indirect interaction. The RKKY interaction, in its usual formulation, provides the hole-mediated static polarization of the ions. To a positive sign of the interaction corresponds a minimum-energy configuration where the hole spin is directed in the opposite direction with respect to the adjacent ions' spins. This effect selects a preferred spin-alignment direction and does not involve a dynamical spin exchange between the holes and the magnetic ions. In our many-particle expansion the static RKKY interaction appears through a second-order bubble diagram (which is not reported here); the third-order diagram represents the dynamical correspondent to the static RKKY interaction. Many authors have pointed out the important role played by the ion-ion RKKY interaction as being an important mechanism which governs the presence or absence of long-ranged magnetic ordering, for example, in connection with systems for which the strength of the ferromagnetic and antiferromagnetic interactions can be modified by varying the vacancy concentration of the host lattice. For instance, $\mathrm{Mn}$ doped $\mathrm{ZnO}$ has antiferromagnetic interactions which can be turned to ferromagnetic interactions in the presence of $\mathrm{Zn}$ vacancies. ${ }^{22,23}$

Furthermore we note that the explicit energy dependence of the density of states influences remarkably the analyticity properties of $\Pi$. In fact $\Pi$ is an integrable function for a three-dimensional (3D) hole systems (where $\rho^{3 D} \sim \sqrt{E}$ ) while it show an infrared nonintegrable divergence for a twodimensional (2D) system when the argument $\xi$ goes to zero and in particular we have that $\lim _{\xi \rightarrow 0} \mathcal{F}_{s_{1}}(\xi) \sim \log (\xi)$. This can be seen explicitly for $T=0$ : in the $3 \mathrm{D}$ case we have

$$
\begin{aligned}
\mathcal{F}_{s_{1}}^{0}(\xi)= & \lim _{\beta \rightarrow \infty} \mathcal{F}_{s_{1}}(\xi)=\rho^{3 D} \theta\left(E_{s_{1}}-\mu_{s_{1}}\right) \\
& \times\left\{\begin{array}{l}
2 \sqrt{E_{s_{1}}-\mu_{s_{1}}}-\sqrt{\xi} \ln \left(\frac{\sqrt{E_{s_{1}}-\mu_{s_{1}}}+\sqrt{\xi}}{\sqrt{E_{s_{1}}-\mu_{s_{1}}}-\sqrt{\xi}}\right), \quad \xi>0 \\
2 \sqrt{E_{s_{1}}-\mu_{s_{1}}}-\sqrt{\xi} \tan ^{-1}\left(\frac{\sqrt{E_{s_{1}}-\mu_{s_{1}}}}{\sqrt{\xi}}\right), \quad \xi<0,
\end{array}\right.
\end{aligned}
$$

which is regular in $\xi=0$, and the divergence in $E_{s_{1}}-\mu_{s_{1}}$ disappears for $T>0$. For a 2D system,

$$
\mathcal{F}_{s_{1}}^{0}(\xi)=\rho^{2 D} \theta\left(E_{s_{1}}-\mu_{s_{1}}\right) \log \left|\frac{\xi-\left(E_{s_{1}}-\mu_{s_{1}}\right)}{\xi}\right|,
$$

and the singularity is not removed for $T>0$.

The presence of a singularity (which is typically defined as an infrared divergence) in the description of the ion-hole scattering processes generally rises when a high-order perturbative expansion is used to treat the Kondo interaction. In the classical formulation of the Kondo problem, the infrared divergence is strictly connected with the presence of a high density of states in correspondence to the Fermi level. ${ }^{1,24}$ In a doped semiconductor device, the Fermi level is located near the band edge and the density of states strongly depends on the dimensionality of the gas carriers. Based on this simple consideration we observe that the hole spin evolution in a DMS exhibits a strong similarity with the standard Kondo's problem in the case of a 2D hole gas for which the density of states is constant. To the contrary, in our model, a regular behavior of the polarization propagator is expected in the limit of a 3D hole gas for which the singularity of the denominator of $\mathcal{F}$ is compensated by the vanishing of the density of states in correspondence to the band edge. The semiconductor host lattice of a DMS provides the interesting property that the many-particle spin interaction becomes stronger when the thickness of the quantum confinement is reduced. In the limit of a two-dimensional hole gas the holeion spin interaction reduces to the standard Kondo interaction. Logarithmic divergences of the polarization propagator are found also in different contexts. For example, in the framework of the drone theory, the Kondo interaction gives rise to a third-order singular scattering in which the electrondrone Green's propagator diverges when the particle energy approaches the Fermi level. ${ }^{25}$ Nonperturbative self-consistent renormalization techniques are applied to treat the unphysical pole corresponding to the Kondo temperature and are able, for example, to reproduce the low resistivity behavior of copper-iron alloy. ${ }^{26} \mathrm{~A}$ similar problem has been also found in the context of the singular behavior of the x-ray photoemission of a metal where a core electron is injected in the conduction band leaving a "deep hole" which strongly interacts with the Fermi gas. In this case, a logarithmic divergence appears in the limit of $t / \varepsilon_{B}$ going to infinity, where $t$ is the time and $\varepsilon_{B}$ is the conduction bandwidth.

In our problem the standard renormalization technique can be used to deal with systems of reduced dimensionality. For the sake of clearness we describe the renormalization procedure applied to the function $\Pi^{b}$; the other terms can be treated similarly. By summing over the tadpole diagrams we obtain the new polarization propagator ${ }^{27}$ 


$$
\begin{gathered}
\Pi_{m^{\prime}, m, s^{\prime}, s}^{*}\left(k^{\prime}, k\right)=\sum_{n=1}^{\infty} \sum_{m_{i}, s_{i}, k_{i}} \underbrace{\Pi_{m^{\prime}, m_{1}, s^{\prime}, s_{1}}\left(k^{\prime}, k_{1}\right) \Pi_{m_{1}, m_{2}, s_{1}, s_{2}}\left(k_{1}, k_{2}\right) \cdots \Pi_{m_{n-1}, m, s_{n-1}, s^{\prime}}\left(k_{n-1}, k\right)}_{n}, \\
\Pi_{m^{\prime}, m, s^{\prime}, s}\left(k^{\prime}, k\right) \equiv \sum_{\eta, \eta^{\prime}} \delta_{\eta, \eta^{\prime}} \Pi_{\mathbf{m}^{\prime}, \mathbf{m}, \mathbf{s}^{\prime}, \mathbf{s}^{\prime}}^{e^{i R_{\eta}\left(k-k^{\prime}\right)}=\Xi_{m^{\prime}, m, s^{\prime}, s}\left(E_{k}\right) \delta\left(E_{k}-E_{k^{\prime}}+\Delta_{m, s^{\prime}}^{m^{\prime}, s}\right)},
\end{gathered}
$$

where we write explicitly the delta term present in the definition of $\Pi^{b}$ and consequently $\Xi$ can be deduced from Eq. (15) and we defined $m_{0}=m^{\prime}, m_{i}=m_{1}, m_{2}, \ldots, m_{n-1}$ and the same for $k_{i}, s_{i}$. After some algebra we obtain (the details of the calculation are given in the Appendix)

$$
\begin{aligned}
\Pi_{m^{\prime}, m, s^{\prime}, s}^{*}\left(k^{\prime}, k\right)= & \delta_{\bar{s}, s^{\prime}} \delta\left(E_{k}-E_{k^{\prime}}+\Delta_{m, s^{\prime}}^{m^{\prime}, s}\right) \\
& \times \frac{\Xi_{\bar{s}, s}\left(E_{k}\right)}{1-\rho^{2}\left(E_{k}\right) \Xi_{s, \bar{s}}\left(E_{k}-\Delta_{s}^{\bar{s}}\right) \Xi_{\bar{s}, s}\left(E_{k}\right)},
\end{aligned}
$$

where $\bar{s} \equiv-s, \Delta_{s^{\prime}}^{s}=E_{s}-E_{s^{\prime}}+E_{m}-E_{m+s g n(s)}$, and to ease notation we define $\Xi_{s^{\prime}, s}=\Xi_{m^{\prime}, m, s^{\prime}, s^{\prime}}$.

\section{NUMERICAL RESULTS}

The present work focuses on a III-V semiconducting host, $\mathrm{GaAs}$, and a magnetic element, $\mathrm{Mn}$, in the low concentration regime. In particular, we consider a sample consisting of a magnetic layer with a Mn concentration of 5\% deposited on a GaAs buffer layer and a semi-insulating GaAs substrate. The background hole density is chosen to be $10^{20} \mathrm{~cm}^{-3}$.

The study of the time evolution of the total magnetization in a GaMnAs DMS for magnetic layers thickness on the order of a few hundred nanometers has been given in Ref. 8 . In such a large system the hole confinement provided by the semi-insulating substrate has no important influence on the spin interaction and, for our purposes, one can safety assume that this configuration corresponds to a nearly-free 3D hole gas. The analysis of the polarization propagator given in Sec. II $\mathrm{C}$ has shown that the third-order corrections to the ion correlations are mainly important for a 2D hole gas. Therefore, in the present section, our attention is focused on the study of a DMS with tiny magnetic layers (2D DMS). Equations (8)-(10) with Eq. (17) have been solved in order to study the role played by the third-order corrections on the time evolution of this sample after laser irradiation.

The initial hole and ion distributions are calculated using the stationary mean-field Zener model. ${ }^{28}$ The laser pulse excitation is assumed to take place at $t=0^{+}$and according to the discussion of Ref. 6 its main effect is to rise instantaneously the initial temperature of the hole gas. Details and justifications of the laser heating processes based on physical grounds are given in Refs. 8 and 29. Let us note that the band parameters of a confined system can be very different from those of the same material in the bulk. Modifications of the optical and transport properties of confined semiconductor materials are due to various effects the most important ones being the stress field and the quantification of the band state along the growth direction. Since in the present work we are interested to highlight the effect of the renormalization procedure on the polarization propagator $\Pi^{*}$, we have used an oversimplified description of the band structure consisting of a single nondegenerate parabolic heavy-hole band. The inclusion of the light hole band in our formalism is straightforward but the computational cost would increase heavily. Theoretical studies have proved that in an unstrained semiconductor the heavy-hole-ion interaction is stronger than the light-hole-ion one. ${ }^{30,31}$ Furthermore, the confinement in a GaAs quantum well (which is considered in the present work) strongly reduces the maximum of the light-hole band, so that its effect on the magnetization dynamics can be neglected (see, for example, Ref. 32). In Ref. 17 the role played by the quantum confinement and the band structure on the magnetization dynamics is analyzed. The mini-band structure has been computed by using a microscopic approach and only second-order effects in the spin-exchange interaction have been included. A forthcoming publication will be devoted to the inclusion of both third-order correlation effects and the detailed band-structure calculation for a 2D DMS.

In Fig. 2 we compare the numerical solution of a secondorder model with respect to the third-order model of Eqs. (8)-(10). We represent the time evolution of the differential magnetization $\delta M$ defined as $\delta M(t) \equiv\left[M_{\text {tot }}(t)\right.$ $\left.-M_{t o t}(0)\right] / M_{t o t}(0)$, where $M_{t o t}=\left\langle M_{z}\right\rangle N^{M}+\left\langle S_{z}\right\rangle N^{h}$ with $\left\langle M_{z}\right\rangle$ $=\Sigma_{m} m \mathfrak{n}_{m}$ and $\left\langle S_{z}\right\rangle=\Sigma_{s} s n_{s}$ as the mean values of the ion and hole spins, respectively. The initial temperatures of the system before the laser excitation are $50 \mathrm{~K}$ [Fig. 2(a)] and $30 \mathrm{~K}$
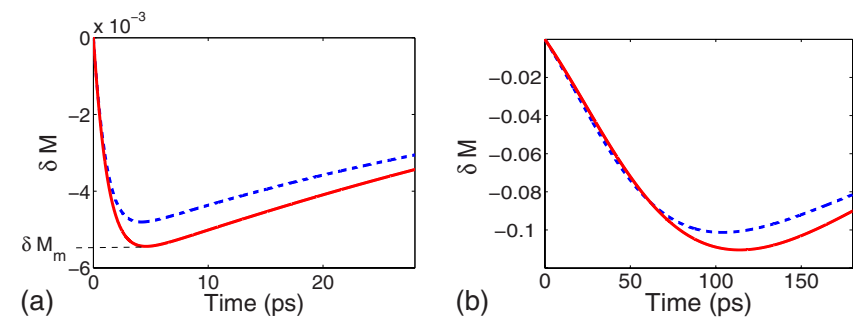

FIG. 2. (Color online) Time evolution of the normalized quantity $\delta M(t) \equiv\left[M_{t o t}(t)-M_{t o t}(0)\right] / M_{t o t}(0)$. The dashed (continuous) line represents the solution obtained from a second-(third-) order approximation of the polarization propagator. The hole background density is $n^{h}=10^{20} \mathrm{~cm}^{-3}$ and the initial temperatures $T^{h}$ are (a) $T^{h}$ $=50 \mathrm{~K}$ and (b) $T^{h}=30 \mathrm{~K}$. 


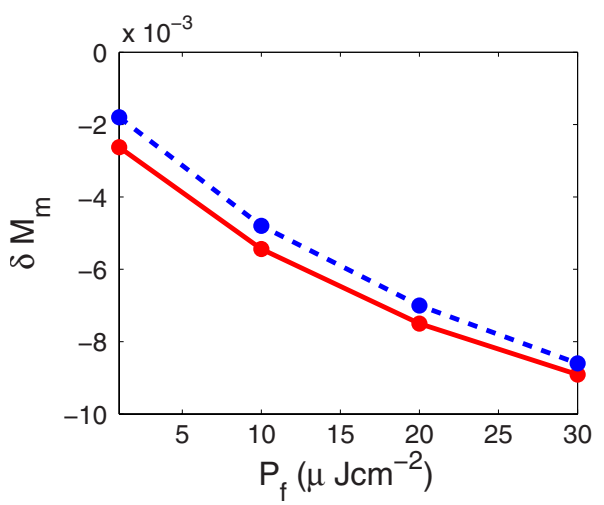

FIG. 3. (Color online) $\delta M_{m}$ as a function of the laser fluence. The dashed (continuous) line represents the solution obtained from a second- (third-) order approximation of the polarization propagator. The quantity $\delta M_{m}$ is defined in Fig. 2(a).

[Fig. 2(b)] and the laser fluence $P_{f}$ is $10 \mu \mathrm{J} \mathrm{cm}$.

Our simulations show that after the laser excitation, the magnetic impurities strongly interact with the out-ofequilibrium hole gas and a redistribution of the spin polarization is obtained. In particular, since the photoexcited holes experience efficient spin-spin scattering with the localized Mn magnetic moments, a net spin polarization is transferred from the ion impurities toward the hole system. This polarization is efficiently dissipated via the spin-orbit coupling, which leads to a net decrease in the total spin magnetization. During this ultrafast demagnetization regime the hole gas loses its energy via carrier-phonon scattering and the hole and ion spins begin to align, allowing the system to recover its initial ferromagnetic order.

The numerical simulations reveal that the third-order terms give a correction to $\delta M$ on the order of $10 \%$. This is a non-negligible contribution to the macroscopical time evolution of the total magnetization which can be, in principle, experimentally measured.

As pointed out in Ref. 6 the time evolution of the magnetization of a strongly out-of-equilibrium excited DMS can be affected by "spin bottleneck," which is a phenomenon where the characteristic time of the demagnetization processes is reduced due to the lack of an efficient microscopic spin transfer between the ions and the hole gas. Our findings show that the inclusion of a further mechanism of spin exchange between the ions and the holes as the third-order Kondo exchange processes, partially reduces (or compensates) the spin bottleneck observed in similar structures.

Finally, in Fig. 3 we report the minimum of the differential magnetization $\delta M_{m}$ for different values of the laser fluence and obtained at second-order (dashed line) and thirdorder (continuous line) levels of approximation. The simulations show that the relative correction becomes less and less important when the power of the laser increases. As shown in Eq. (15), one of the relevant differences between the one- site (second- and third-order terms) and the two-site third-order terms is the RKKY- like form of the latter, where the sinus of the product between the hole momentum and the mean ion-ion distance is present in the integral. When the laser power increases, the hole states with high momentum are more and more populated and, due to the oscillating form of the interaction, the contribution of these high-energy states tends to cancel, justifying a relative lowering of the two-site third-order terms. Furthermore, as already pointed out, also the sign of the third-order interaction given in Eq. (15) depends on the mean distance between two nearest magnetic ions. In particular, one can expect a completely different behavior of the third-order contribution if a disordered system with dislocations or $\mathrm{Mn}$ interstitial inclusions is investigated. The numerical complexity related to the numerical solution of Eqs. (8)-(10) prevents us to apply our model to highly nonhomogeneous structures.

\section{CONCLUSIONS}

Ultrafast magnetization dynamics in diluted magnetic semiconductors is investigated using a theoretical model based on the pseudofermion formalism and a third-order many-particle expansion of the Kondo spin-exchange interaction. Differing from the standard RKKY approach, our theoretical methodology does not rely on crude simplifications and hypothesis in the description of the hole state which is treated here in a out-of-equilibrium regime. Dynamical RKKY-like interactions and double-exchange mechanism based on the exact Kondo interaction emerge naturally from the pseudofermion approach. Furthermore, our method is able to treat dynamically particle-particle correlations beyond the mean-field approximation and provides a theoretical framework allowing the inclusion of a detailed description of materials with complex chemical composition or/and with complex magnetic ordering such as antiferromagnets and spin glasses. The analysis of the third-order Green's function reveals that the many-particle expansion is not generally well defined and an infrared Kondo-like divergence can occur. In particular, the bare polarization propagator fails to converge for a two-dimensional DMS and a regular behavior is found for 3D systems. As a final result, our approach predicts an enhancement of the spin-spin correlation in the case of reduced-dimensionality systems. Numerical simulations have been performed on GaMnAs and show that dynamical many-particle correlations play a significant role in the time evolution of the total magnetization. Therefore, we conclude by saying that dynamical correlations studied here should play an important role in the ultrafast magnetization dynamics observed with pump-probe magneto-optical spectroscopy.

\section{ACKNOWLEDGMENT}

We acknowledge financial support from the French National Research Agency ANR (Project No. ANR-06-BLAN0059).

\section{APPENDIX: RENORMALIZATION OF II}

A detailed derivation of Eq. (17) is given here. Let us consider a spin-flip process assuming $m^{\prime} \neq m$ and $s^{\prime}=-s$. To ease notation we define $\Pi_{s^{\prime}, s}\left(k^{\prime}, k\right) \equiv \Pi_{m^{\prime}, m, s^{\prime}, s}\left(k^{\prime}, k\right)$, $\Xi_{s^{\prime}, s}$ $\equiv \Xi_{m^{\prime}, m, s^{\prime}, s}$, and 


$$
\widetilde{\Pi}_{s_{i}, s_{i+2}}\left(k_{i}, k_{i+2}\right) \equiv \sum_{s_{i+1}, k_{i+1}} \Pi_{s_{i}, s_{i+1}}\left(k_{i}, k_{i+1}\right) \Pi_{s_{i+1}, s_{i+2}}\left(k_{i+1}, k_{i+2}\right)=\widetilde{\Xi}_{s_{i}, s_{i+2}}\left(k_{i}, k_{i+2}\right) \delta\left(E_{k_{i}}-E_{k_{i+2}}\right) \delta_{s_{i}, s_{i+2}},
$$

with

$$
\widetilde{\Xi}_{s_{i}, s_{i+2}}\left(k_{i}, k_{i+2}\right) \equiv \rho\left(E_{k_{i}}+\Delta_{s_{i}}^{\bar{s}_{i}}\right) \Xi_{s_{i}, s_{i}}\left(E_{k_{i}}\right) \Xi_{\bar{s}_{i}, s_{i}}\left(E_{k_{i}}+\Delta_{s_{i}}^{\overline{s_{i}}}\right),
$$

where $\bar{s} \equiv-s$ and $\Delta_{s^{\prime}}^{s}=E_{s}-E_{s^{\prime}}+E_{m}-E_{m+\operatorname{sgn}(s)}$. We separately consider the parts with an even or odd number of factors. Thus, we have

$$
\begin{aligned}
\Pi_{m^{\prime}, m, s^{\prime}, s}^{*}\left(k^{\prime}, k\right)= & \Pi_{s^{\prime}, s}\left(k^{\prime}, k\right)+\sum_{n=3,5, \ldots} \sum_{s_{i}, k_{i}} \underbrace{\Pi_{s^{\prime}, s_{1}}\left(k^{\prime}, k_{1}\right) \tilde{\Pi}_{s_{1}, s_{3}}\left(k_{1}, k_{3}\right) \tilde{\Pi}_{s_{3}, s_{5}}\left(k_{3}, k_{5}\right) \cdots \tilde{\Pi}_{s_{n-2}, s}\left(k_{n-2}, k\right)}_{n=2,4, \ldots} \\
& +\underbrace{\infty}_{(n-1) / 2} \underbrace{\tilde{\Pi}_{s^{\prime}, s_{2}}\left(k^{\prime}, k_{2}\right) \tilde{\Pi}_{s_{2}, s_{4}}\left(k_{2}, k_{4}\right) \cdots \tilde{\Pi}_{s_{n-2}, s}\left(k_{n-2}, k\right)}_{n / 2} \\
= & \delta_{\bar{s}, s^{\prime}} \delta\left(E_{k}-E_{k^{\prime}}+\Delta_{m, s^{\prime}}^{m^{\prime}, s}\right) \frac{\Xi_{\bar{s}, s}\left(E_{k}\right)}{1-\rho^{2}\left(E_{k}\right) \Xi_{s, s}\left(E_{k}-\Delta_{s}^{s}\right) \Xi_{\bar{s}, s}\left(E_{k}\right)}+\delta_{s, s^{\prime}} \delta\left(E_{k}-E_{k^{\prime}}\right) \sum_{n=1}^{\infty}\left[\rho\left(E_{k}\right) \widetilde{\Xi}_{s, s}(k, k)\right]^{n} .
\end{aligned}
$$

The second term gives no contribution to $\mathcal{W}$ and we finally obtain

$$
\Pi_{m^{\prime}, m, s^{\prime}, s}^{*}\left(k^{\prime}, k\right)=\delta_{\overline{s, s^{\prime}}} \delta\left(E_{k}-E_{k^{\prime}}+\Delta_{m, s^{\prime}}^{m^{\prime}, s}\right) \frac{\Xi_{\bar{s}, s}\left(E_{k}\right)}{1-\rho^{2}\left(E_{k}\right) \Xi_{s, \bar{s}}\left(E_{k}-\Delta_{s}^{\bar{s}}\right) \Xi_{\bar{s}, s}\left(E_{k}\right)} .
$$

${ }^{1}$ J. Kondo, Prog. Theor. Phys. 32, 37 (1964).

${ }^{2}$ S. Doniach and E. H. Sondheimer, Green's Functions for Solid State Physicists (Imperial College Press, London, 1998).

${ }^{3}$ J. Wang, C. Sun, J. Kono, A. Oiwa, H. Munekata, Ł. Cywiński, and L. J. Sham, Phys. Rev. Lett. 95, 167401 (2005).

${ }^{4}$ K. Ohno, S. Ohya, and M. Tanaka, J. Supercond. Novel Magn. 20, 417 (2007)

${ }^{5}$ B. König, I. A. Merkulov, D. R. Yakovlev, W. Ossau, S. M. Ryabchenko, M. Kutrowski, T. Wojtowicz, G. Karczewski, and J. Kossut, Phys. Rev. B 61, 16870 (2000).

${ }^{6}$ Ł. Cywiński and L. J. Sham, Phys. Rev. B 76, 045205 (2007).

${ }^{7}$ O. Morandi, P.-A. Hervieux, and G. Manfredi, Eur. Phys. J. D 52, 155 (2009).

${ }^{8}$ O. Morandi, P.-A. Hervieux, and G. Manfredi, New J. Phys. 11, 073010 (2009).

${ }^{9}$ M. D. Kapetanakis and I. E. Perakis, Phys. Rev. Lett. 101, 097201 (2008).

${ }^{10}$ A. Singh, S. K. Das, A. Sharma, and W. Nolting, J. Phys.: Condens. Matter 19, 236213 (2007).

${ }^{11}$ B. Skubic, J. Hellsvik, L. Nordstrom, and O. Eriksson, J. Phys.: Condens. Matter 20, 315203 (2008).

${ }^{12}$ J. Hellsvik, B. Skubic, L. Nordstrom, B. Sanyal, O. Eriksson, P. Nordblad, and P. Svedlindh, Phys. Rev. B 78, 144419 (2008).

${ }^{13}$ A. A. Abrikosov, Physics (Long Island City, N.Y.) 2, 5 (1965).

${ }^{14}$ P. Coleman, Phys. Rev. B 28, 5255 (1983).

${ }^{15}$ O. Morandi, P.-A. Hervieux, and G. Manfredi, Nuovo Cimento Soc. Ital. Fis., C 33, 173 (2010).

${ }^{16}$ E. K. U. Gross, E. Runge, and O. Heinonen, Many-Particle Theory (Adam Hilger, Bristol, 1991).

${ }^{17}$ O. Morandi, P.-A. Hervieux, and G. Manfredi, Phys. Rev. B 81,
155309 (2010).

${ }^{18}$ R. Bouzerar, G. Bouzerar, and T. Ziman, Phys. Rev. B 73, 024411 (2006).

${ }^{19}$ P. M. Krstajić, F. M. Peeters, V. A. Ivanov, V. Fleurov, and K. Kikoin, Phys. Rev. B 70, 195215 (2004).

${ }^{20}$ D. J. Priour, E. H. Hwang, and S. Das Sarma, Phys. Rev. Lett. 92, 117201 (2004).

${ }^{21}$ H. Haug and A.-P. Jauho, Quantum Kinetics in Transport and Optics of Semiconductors, Springer Series in Solid-State Sciences (Springer, New York, 1996).

${ }^{22}$ D. Iuşan, B. Sanyal, and O. Eriksson, Phys. Rev. B 74, 235208 (2006).

${ }^{23}$ L. Bergqvist, O. Eriksson, J. Kudrnovský, V. Drchal, P. Korzhavyi, and I. Turek, Phys. Rev. Lett. 93, 137202 (2004).

${ }^{24}$ R. D. Mattuck, A Guide to Feynman Diagrams in the ManyBody Problem (Dover, New York, 1976).

${ }^{25}$ D. C. Mattis, Theory of Magnetism (Dover, New York, 1965).

${ }^{26}$ Y. Nagaoka, Phys. Rev. 138, A1112 (1965).

${ }^{27}$ A. L. Fetter and J. D. Walecka, Quantum Theory of ManyParticle Systems (McGraw-Hill, New York, 1971).

${ }^{28}$ N. Kim, H. Kim, J. W. Kim, S. J. Lee, and T. W. Kang, Phys. Rev. B 74, 155327 (2006).

${ }^{29}$ J. Wang, I. Cotoros, K. M. Dani, X. Liu, J. K. Furdyna, and D. S. Chemla, Phys. Rev. Lett. 98, 217401 (2007).

${ }^{30}$ K. A. Kikoin and V. N. Fleurov, Transition Metal Impurities in Semiconductors (World Scientific, Singapore, 1994).

${ }^{31}$ T. Dietl, H. Ohno, and F. Matsukura, Phys. Rev. B 63, 195205 (2001).

${ }^{32}$ T. Jungwirth, J. Konig, J. Sinova, J. Kucera, and A. H. MacDonald, Phys. Rev. B 66, 012402 (2002). 